\title{
ON MIXED VOLUMES OF NONCONVEX SETS
}

\section{WOLFGANG WEIL}

ABSTRACT. Mixed volumes have been extended nonconvex sets by several authors. It is shown that in any such extension at least one essential property of mixed volumes is lost.

Introduction. Investigating the interrelations between volume and vector addition of convex bodies in Euclidean $d$-space $E^{d}$, one is led, in a very natural way, to the notion of mixed volume, which is fundamental in the Brunn-Minkowski theory of convex bodies. For nonconvex subsets of $E^{d}$ the connections between measurability and Lebesgue measure on one hand, and vector addition on the other, seem to be much less clear-cut, as is indicated, for instance, by some examples due to Sierpinski [6] and Henstock-Macbeath [5]. However, natural extensions of mixed volumes to certain nonconvex sets have been proposed in the literature, see Geppert [4], Blaschke [2], BerwaldVarga [1]. The purpose of this note is to show that in any such extension the mixed volume necessarily loses at least one of its essential properties.

I wish to thank Professor R. Schneider for several useful suggestions.

1. Statement of the Theorem. Let $\mathbf{H}$ denote the set of all convex bodies in $E^{d}$ and let $\mathbf{H}^{d}$ be the dofold Cartesian product. For $K, L \subset E^{d}$ the vector sum $K+L$ is defined by $K+L=\left\{z \in E^{d} \mid z=x+y, x \in K, y \in L\right\}$. For $M \subset E^{d}$ let $\mathbf{H}_{M}$ be the set of all sum-sets $L+K, K \in \mathbf{H}, L=M$ or $\{0\}$, and let $\mathbf{H}_{M}^{d}$ be the $d$-fold Cartesian product. conv $M$ and bd $M$ denote the convex hull and the boundary of the set $M \subset E^{d} \cdot \lambda$ is the Lebesgue measure. The multiple $\alpha M, a \geq 0$, of a set $M \subset E^{d}$ is defined in the usual manner, $\alpha M=$ $\left\{z \in E^{d} \mid z=\alpha x, x \in M\right\}$.

The mixed volume $V$ is a real-valued function on $\mathbf{H}^{d}$ which is uniquely determined by the following three properties (see [3]):

(1) $V(K, \ldots, K)=\lambda(K)$ for all $K \in \mathbf{H}$,

(2) $V$ is additive in each variable, that is

$$
\begin{aligned}
V\left(K_{1}, \ldots, K_{j-1}, K_{j}\right. & \left.+K_{j}^{\prime}, K_{j+1}, \ldots, K_{d}\right) \\
= & V\left(K_{1}, \ldots, K_{j-1}, K_{j}, K_{j+1}, \ldots, K_{d}\right) \\
& +V\left(K_{1}, \ldots, K_{j-1}, K_{j}^{\prime}, K_{j+1}, \ldots, K_{d}\right),
\end{aligned}
$$

Received by the editors April 10, 1974.

AMS (MOS) subject classifications (1970). Primary 52A20.

Key words and phrases. Convex body, mixed volume, vector addition, compact sets.

Copyright $\odot$ 1975, American Mathematical Society 
for all $K_{1}, \ldots, K_{j-1}, K_{j}, K_{j}^{\prime}, K_{j+1}, \ldots, K_{d} \in \mathbf{H}$, and all $j \in\{1, \ldots, d\}$,

(3) $V$ is symmetric in all variables.

Other properties, such as nonnegativity, continuity, monotony, etc. can be derived from (1), (2), and (3).

Trivially, the mixed volume can be extended to $\mathbf{H}_{M}^{d}$ for certain measuro able, nonconvex subsets $M$ of sets $K \in \mathbf{H}$ with $\lambda(M)=\lambda(K)$. Also, an extension is possible if $M$ is unbounded and $V$ is allowed to take the value $+\infty$. Therefore, we focus our attention on compact sets $M$.

Theorem. Let $M$ be a nonvoid, compact subset of $E^{d}$ and $V$ a real. valued function on $\mathbf{H}_{M}^{d}$ with the following two properties:

(4) $V(L, \ldots, L)=\lambda(L)$ for all $L \in \mathbf{H}_{M}$,

(5) $V$ is additive in each variable.

Then $M$ is a convex body.

Let us remark that, by multilinear extension, mixed volumes can be deo fined for elements of the vector space generated by the cone $\mathbf{H}$ (see [7]). This is a useful method to prove properties of convex bodies. However, it is not possible to find a geometric representation of differences of convex bodies. The definition of supportable sets in [4] seems to be a natural interpretation of certain differences of convex bodies, but the addition which is used in [4] and which is induced by the addition of the support functions is not vector addition.

2. Addition of convex hulls. The proof of the theorem is based on the following two results which state that, starting from a compact set $M$, we can get a convex one by adding a certain multiple of the convex hull of $M$. For properties of convex bodies which we use in the following we refer to [3].

Proposition 1. For $K \in \mathbf{H}$ we have $K+\mathrm{bd} K=2 K$.

Proof. As is well known, convex sets $K$ satisfy $K+K=2 K$. Thus, we have $K+b d K \subset 2 K$. On the other hand, for $x \in K$, let $l(x)$ be the line through $x$ and the origin (or any line through $x$ if $x$ is the origin). The intersection $l(x) \cap K$ is a segment $\left[x_{1}, x_{2}\right]=\operatorname{conv}\left\{x_{1}, x_{2}\right\}$ with suitable $x_{1}$, $x_{2} \in E^{d}$. Because of $l(x) \cap 2 K=2\left[x_{1}, x_{2}\right]$ and $l(x) \cap \mathrm{bd} K=\left\{x_{1}, x_{2}\right\}$ we derive from

$$
\left[x_{1}, x_{2}\right]+\left\{x_{1}, x_{2}\right\}=2\left[x_{1}, x_{2}\right]
$$

that $x+x \in K+$ bd $K$. But (6) follows by an easy computation.

Proposition 2. For compact $M \subset E^{d}$ we have

$$
M+\left(2^{d}-1\right) \operatorname{conv} M=2^{d} \operatorname{conv} M \text {. }
$$

Proof. We use induction. The assertion is obvious for $d=1$ in view of Proposition 1. 
For $d>1$ we consider the set $M^{\prime}=M+\left(2^{d-1}-1\right)$ conv $M$, which is compact. For a compact set $L \subset E^{d}$ and a unit vector $u \in E^{d}$ the support set $L(u)$ is defined by $L(u)=E(u) \cap L$, where $E(u)$ is the supporting hyperplane of $L$ with outer unit normal $u$. As is well known, the support sets of a vector sum equal the vector sums of the corresponding support sets. Applied to $M^{\prime}$, this yields

$$
M^{\prime}(u)=M(u)+\left(2^{d-1}-1\right)(\operatorname{conv} M)(u)
$$

By the definition of the convex hull we have $(\operatorname{conv} M)(u)=\operatorname{conv} M(u) . M(u)$ has dimension at most $d-1$ and hence, by the inductive assumption,

$$
M^{\prime}(u)=M(u)+\left(2^{d-1}-1\right) \operatorname{conv} M(u)=2^{d-1} \operatorname{conv} M(u) .
$$

Thus, if we form the union over all unit vectors $u$,

$$
\bigcup M^{\prime}(u)=2^{d-1} \mathrm{bd}(\operatorname{conv} M),
$$

and because of $M+\left(2^{d}-1\right) \operatorname{conv} M=M^{\prime}+2^{d-1}$ conv $M$, we get

$$
\begin{gathered}
2^{d-1} \operatorname{conv} M+2^{d-1} \text { bd }(\operatorname{conv} M) \subset M+\left(2^{d}-1\right) \operatorname{conv} M \\
\subset \operatorname{conv} M+\left(2^{d}-1\right) \operatorname{conv} M=2^{d} \operatorname{conv} M .
\end{gathered}
$$

In view of Proposition 1 we conclude $M+\left(2^{d}-1\right) \operatorname{conv} M=2^{d} \operatorname{conv} M$.

3. Proof of the Theorem. We show that

$$
V(M, \ldots, M)=V(\underbrace{\operatorname{conv} M, \ldots, \operatorname{conv} M}_{j}, \frac{M, \ldots, M}{d-j})
$$

for all $j \in\{0, \ldots, d\}$. Suppose (7) is true for a $j \in\{0, \ldots, d-1\}$. We show that it is true for $j+1$, using (5) and Proposition 2:

$$
\begin{aligned}
& V(M, \ldots, M)=V(\underbrace{\operatorname{conv} M, \ldots, \operatorname{conv} M}_{j}, \frac{M, \ldots, M}{d-j}) \\
& =V(\underbrace{\operatorname{conv} M, \ldots, \operatorname{conv} M,}_{j} M+\left(2^{d}-1\right) \operatorname{conv} M, \frac{M, \ldots, M)}{d-j-1} \\
& -V(\underbrace{\operatorname{conv} M, \ldots, \operatorname{conv} M,}_{j}\left(2^{d}-1\right) \operatorname{conv} M, \frac{M, \ldots, M)}{d-j-1} \\
& =V(\underbrace{\operatorname{conv} M, \ldots, \operatorname{conv} M,}_{j} 2^{d} \operatorname{conv} M, \frac{M, \ldots, M)}{d-j-1} \\
& -V(\underbrace{\operatorname{conv} M, \ldots, \operatorname{conv} M}_{j},\left(2^{d}-1\right) \operatorname{conv} M, \frac{M, \ldots, M)}{d-j-1} \\
& =V(\underbrace{\operatorname{conv} M, \ldots, \operatorname{conv} M}_{j+1}, \frac{M, \ldots, M)}{d-j-1} \text {. }
\end{aligned}
$$


In case $j=d,(7)$ implies, in view of $(4), \lambda(M)=\lambda(\operatorname{conv} M)$. As $M$ is compact we conclude $M=$ conv $M$, provided $\lambda(M) \neq 0$. If $\lambda(M)=\lambda(\operatorname{conv} M)=0$, we take a lower dimensional cube $C$ orthogonal to $M$ such that $M^{\prime}=M+C$ has full dimension. Repeating the proof for $M^{\prime}$, we conclude $\lambda\left(M^{\prime}\right)=\lambda\left(\operatorname{conv} M^{\prime}\right)$ $\neq 0$. Thus, $M+C=M^{g}=\operatorname{conv} M^{\prime}=\operatorname{conv} M+C$, which implies $M=\operatorname{conv} M$. This completes the proof of the Theorem.

\section{REFERENCES}

1. L. Berwald and O. Varga, Integralgeometrie 24. Über die Schiebungen im Raum, Math. Z. 42 (1937), 710-736.

2. W. Blaschke, Integralgeometrie 21. Über Schiebungen, Math. Z. 42 (1937), $399-410$.

3. T. Bonnesen and W. Fenchel, Theorie der konvexen Körper, Springer, Berlin, 1934; reprint, Chelsea, New York, 1948.

4. H. Geppert, Über den Brunn-Minkouskischen Satz, Math. Z. 42 (1937), 238254.

5. R. Henstock and A. M. Macbeath, On the measure of sumosets. I: The theorems of Brunn, Minkowski, and Lusternik, Proc. London Math. Soc. (3) 3 (1953), 182-194. MR 15, 109.

6. W. Sierpinski, Sur la question de la measurabilité de la base de M. Hamel, Fund. Math. 1 (1920), 105-111.

7. W. Weil, Über den Vektorraum der Differenzen von Stützfunktionen konvexer Körper, Math. Nachr. 59 (1974), 353-368.

FACHBEREICH MATHEMATIK, TECHNISCHE UNIVERSITÄT BERLIN, BERLIN, WEST GERMANY

Current address: Mathematisches Institut der Albert-Ludwigs-Universität, 7800 Freiburg i. Br., Hebelstrasse 40, West Germany 